\title{
BOUNDARY INTEGRABLE QUANTUM FIELD THEORIES
}

\author{
PATRICK DOREY \\ Department of Mathematical Sciences, University of Durham, \\ Durham DH1 3LE, UK \\ E-mail: p.e.dorey@dur.ac.uk
}

\begin{abstract}
A brief survey of recent results in the study of boundary integrable quantum field theories, indicating some currently open problems. Based on lectures given at the 2000 Eötvös Summer School in Physics on "Nonperturbative QFT methods and their applications". DCTP/01/13; hep-th/0101174
\end{abstract}

These lectures concerned the properties of quantum field theories in the presence of boundaries. There are many different approaches to this subject. One can begin by studying conformal field theories with boundaries - the principal theme of the lectures at this school by Jean-Bernard Zuber and by Christoph Schweigert - and then, as described in Gérard Watts' lectures, consider their perturbations. In many cases these perturbations result in massive integrable quantum field theories, and it was the direct study of such theories in their own right that formed my main topic. A number of reviews of this subject can be found on the electronic archives, and so in this contribution I shall restrict myself to an outline of the questions touched on in my talks, and a brief list of references to which the interested reader can turn to find at least some of the answers.

The focus will be on boundary field theories which are integrable, and if the usual locality conditions are also imposed then, just as for theories without boundaries, the dimensionality of space must be restricted to one. With the time dimension remaining infinite, there are then just two possible 'boundary geometries': the theory can be defined either

- on a half-line

or

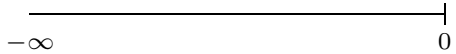

- on a finite interval

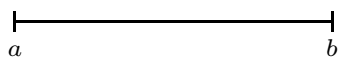

It can then be studied either as a classical, or directly as a quantum field theory. Key questions that one might ask include the following:

(a) For a given $\left\{\begin{array}{c}\text { classical } \\ \text { quantum }\end{array}\right\}$ integrable model on the full line ('in the bulk'), which boundary conditions are compatible with integrability? 
(b) Given a massive boundary integrable quantum field theory, how do bulk particles scatter off the boundary, and how should the 'exact S matrix' technology be generalised to encompass boundary problems?

(c) Can perturbation theory be set up to test any exact predictions?

(d) In the presence of a boundary, the spectrum of bulk excitations may, depending on the boundary condition, be augmented by a number of 'boundary bound states'. How is this spectrum encoded in the amplitudes for the scattering of bulk particles off the boundary?

(e) What does the spectrum of the theory look like on a finite interval?

Before any of these issues can be addressed, the properties of massive integrable quantum field theories on the full line should be understood. The classic reference is the article [1] by Zamolodchikov and Zamolodchikov; a couple of more recent reviews, containing many more references, are [2, 3].

Having digested this material, we can return to the novel questions which arise when the model has a boundary. Much of the recent interest in this topic can be traced to the pioneering work 泪 by Ghoshal and Zamolodchikov. In particular, question (b), concerning the correct generalisation of the ideas of exact $\mathrm{S}$ matrix theory to boundary theories, was answered in reasonably complete detail in this paper. Other early explorations of this point can be found in references [5].

Questions (a) and (d) were also raised in 偖. For the case of the sineGordon model, it was argued on the basis of low-lying conserved quantities that there should be a two-parameter family of classically integrable boundary conditions for each bulk theory. The generalisation of this work to other classical theories, and the development of more sophisticated techniques allowing complete integrability to be established, provides a rich source of open problems. Further work in this area includes [8 12]. To treat question (d), the spectrum of boundary bound states, one has to learn how to interpret the pole structure of the reflection amplitudes. The basic rules were laid out in 跑, while in [13] it was pointed out that the story is sometimes complicated by multiple rescattering processes, the so-called boundary Coleman-Thun mechanism. These methods have been applied to a variety of models 14 16], but it remains an open problem to place them on a solid field-theoretic base.

This leads naturally to question (c), the perturbative treatment of boundary models, and this remains a difficult problem. A framework for calculations has been set up in 17 19, and tested in a number of examples. Particularly tricky are cases involving expansions about non-trivial classical backgrounds; see [10,20 23] for some sample discussions of the issues involved. Recently, progress has also been achieved using WKB techniques. These developments are reviewed in 24]. 
An alternative to perturbation theory as a method to test proposed boundary reflection amplitudes is to study their implications for the spectrum of a theory on a finite interval, question (e) above. Integral equations ('of TBA [Thermodynamic Bethe Ansatz] type') encoding the ground-state energy were first given in 25]. It turned out that these equations are not always correct, and in [26] modifications for situations where they break down were found, along with their generalisations to cover excited states. An important part of this work was the testing of results against a generalisation of the so-called truncated conformal space approach (TCSA) to models with boundaries. Further discussion of these methods, and more references, can be found in 27 29], and in Gérard Watts' contribution to these proceedings. However the number of massive models for which this programme has been completed remains small, and further examples would be welcome. Progress on these issues is also being made from the point of view of lattice models [30].

If the rôles of the space and time directions are swapped over, then the boundary condition is replaced by an initial boundary state, $|B\rangle$ [ [4]. Space now runs from $-\infty$ to $+\infty$, and can be compactified to a circle by imposing periodic boundary conditions. The inner product of $|B\rangle$ with the ground state for the theory on the circle is of particular interest, being related to the so-called $g$ function of Affleck and Ludwig [31]. An alternative set of integral equations, also of TBA type, can be used to study the evolution of this quantity with system size $[32,25,33,34]$. These equations are, however, only fully satisfactory when the bulk theory is massless [33]. For the time being it is an open question whether modifications can be found to describe the flow of the $g$-function in the more general massive situation.

This has been a very rapid tour, giving only a flavour of the literature on boundary integrable quantum field theories. To finish, I shall just mention three further review articles. Reference [35] is recommended for a more detailed treatment of the formal aspects of the subject, while the articles [36, 37] are excellent starting-points for those interested in the applications of boundary integrability to concrete problems in condensed matter physics.

\section{Acknowledgements}

I would like to thank Zalán Horváth and Laci Palla for the invitation to discuss this subject at the Eötvos school, Ed Corrigan, Roberto Tateo and Gérard Watts for helpful comments, and the UK EPSRC for an Advanced Fellowship. I was supported in part by a TMR grant of the European Commission, reference ERBFMRXCT960012. 


\section{References}

1. A.B. Zamolodchikov and Al.B. Zamolodchikov, 'Factorised S-matrices in two dimensions as the exact solutions of certain relativistic quantum field theory models', Ann. Phys. 120 (1979) 253

2. G. Mussardo, 'Off-critical statistical models: factorized scattering theories and bootstrap program', Phys. Rep. 218 (1992) 215

3. P. Dorey, 'Exact S-matrices', in the proceedings of the 1996 Eötvös Graduate School (Springer 1997), hep-th/9810026

4. S. Ghoshal and A.B. Zamolodchikov, 'Boundary $S$ matrix and boundary state in two-dimensional integrable quantum field theory', Int. J. Mod. Phys. A9 (1994) 3841, hep-th/9306002

5. I.V. Cherednik, 'Factorizing particles on a half-line and root systems', Theor. Math. Phys. 61 (1984) 977

6. A. Fring and R. Köberle, 'Factorized scattering in the presence of reflecting boundaries', Nucl. Phys. B421 (1994) 159, hep-th/9304141

7. R. Sasaki, 'Reflection bootstrap relations for Toda field theory', Plenary talk at the International Conference on Interface between Physics and Mathematics IPM93, Hangzhou, China; YITP/U-93-33, hep-th/9311027

8. E. Corrigan, P.E. Dorey, R.H. Rietdijk and R. Sasaki, 'Affine Toda field theory on a half-line', Phys. Lett. B333 (1994) 83, hep-th/9404108

9. A. MacIntyre, 'Integrable boundary conditions for classical sine-Gordon theory', J. Phys. A28 (1995) 1089, hep-th/9410026

10. E. Corrigan, P.E. Dorey and R.H. Rietdijk, 'Aspects of affine Toda field theory on a half line', Prog. Theor. Phys. Suppl. 118 (1995) 143, hep-th/9407148

11. P. Bowcock, E. Corrigan, P.E. Dorey and R.H. Rietdijk, 'Classically integrable boundary conditions for affine Toda field theories', Nucl. Phys. B445 (1995) 469, hep-th/9501098

12. T. Inami, S. Odake and Y-Z. Zhang, 'Supersymmetric extension of the sine-Gordon theory with integrable boundary interactions', Phys. Lett. B359 (1995) 118, hep-th/9506157

13. P. Dorey, R. Tateo and G.M.T. Watts, 'Generalisations of the Coleman-Thun mechanism and boundary reflection factors', Phys. Lett. B448 (1999) 249, hep-th/9810098

14. H. Saleur and S. Skorik, 'Boundary bound states and boundary bootstrap in the sine-Gordon model with Dirichlet boundary conditions', J. Phys. A28 (1995) 6605, hep-th/9502011

15. G. Delius and G.M. Gandenberger, 'Particle reflection amplitudes in 
$A_{N}^{(1)}$ Toda field theories', Nucl. Phys. B554 (1999) 325, hep-th/9904002

16. P. Mattsson and P. Dorey, 'Boundary spectrum in the sine-Gordon model with Dirichlet boundary conditions', J. Phys. A33 (2000) 9065, hep-th/0008071

17. J.D. Kim, 'Boundary reflection matrix in perturbative quantum field theory', Phys. Lett. B353 (1995) 213, hep-th/9504018

18. E. Corrigan, 'On duality and reflection factors for the sinh-Gordon model', Int. J. Mod. Phys. A13 (1998) 2709, hep-th/9707235

19. N. Topor, 'Perturbation method for boundary $S$-matrix in $2 \mathrm{D}$ quantum field theory', Mod. Phys. Lett. A12 (1997) 2951

20. H. Saleur, S. Skorik and N.P. Skorik, 'The boundary sine-Gordon model: classical and semiclassical analysis', Nucl. Phys. B441 (1995) 421, hep-th/9408004

21. P. Bowcock, 'Classical backgrounds and scattering for affine Toda field theory on a half line', JHEP 9805:008, 1998, hep-th/9609233

22. M. Perkins and P. Bowcock, 'Quantum corrections to the classical reflection factor in $a_{2}^{(1)}$ Toda field theory', Nucl. Phys. B358 (1999) 612 , hep-th/9807146

23. G. Delius, 'Restricting affine Toda theory to the half line', JHEP 9809:016, 1998, hep-th/9807189

24. E. Corrigan, 'Boundary bound states in integrable quantum field theory', JHEP proceedings PRHEP-tmr2000/037, hep-th/0010094

25. A. Leclair, G. Mussardo, H. Saleur and S. Skorik, 'Boundary energy and boundary states in integrable quantum field theories', Nucl. Phys. B453 (1995) 581, hep-th/9503227

26. P. Dorey, A. Pocklington, R. Tateo and G.M.T. Watts, 'TBA and TCSA with boundaries and excited states', Nucl. Phys. B525 (1998) 641, hep-th/9712197

27. P. Dorey, M. Pillin, R. Tateo and G.M.T. Watts, 'One point functions in perturbed boundary conformal field theories', Nucl. Phys. B594 (2001) 625, hep-th/0007077

28. K. Graham, I. Runkel and G.M.T. Watts, 'Renormalization group flows of boundary theories', JHEP proceedings PRHEP-tmr2000/040, hep-th/0010082

29. P. Dorey, M. Pillin, A. Pocklington, I. Runkel, R. Tateo and G.M.T. Watts, 'Finite size effects in perturbed boundary conformal field theories', JHEP proceedings PRHEP-tmr2000/035, hep-th/0010278

30. P.A. Pearce, L. Chim and C. Ahn, 'Excited TBA equations I: massive tricritical Ising model', hep-th/0012223 
31. I. Affleck and A.W.W. Ludwig, 'Universal noninteger 'ground state degeneracy' in critical quantum systems', Phys. Rev. Lett. 67 (1991) 161

32. V.V. Bazhanov, S.L. Lukyanov and A.B. Zamolodchikov, 'Integrable structure of conformal field theory, quantum KdV theory and thermodynamic Bethe ansatz', Commun. Math. Phys. 177 (1996) 381, hep-th/9412229

33. P. Dorey, I. Runkel, R. Tateo and G.M.T. Watts, ' $g$-function flow in perturbed boundary conformal field theories', Nucl. Phys. B578 (2000) 85, hep-th/9909216

34. C. Ahn and R.I. Nepomechie, 'The scaling supersymmetric Yang-Lee model with boundary', Nucl. Phys. B594 (2001) 660, hep-th/0009250

35. E. Corrigan, 'Integrable field theories with boundary conditions', talk given at the International Workshop on Frontiers in Quantum Field Theory, Urumqi, P.R. China, 1996, hep-th/9612138

36. H. Saleur, 'Lectures on non perturbative field theory and quantum impurity problems', in the proceedings of the 1998 Les Houches Summer School, cond-mat/9812110

37. H. Saleur, 'Lectures on non perturbative field theory and quantum impurity problems. Part II', cond-mat/0007309 